\title{
A New Versatile Discrete Distribution
}

by Rolf Turner

\begin{abstract}
This paper introduces a new flexible distribution for discrete data. Approximate moment estimators of the parameters of the distribution, to be used as starting values for numerical optimization procedures, are discussed. "Exact" moment estimation, effected via a numerical procedure, and maximum likelihood estimation, are considered. The quality of the results produced by these estimators is assessed via simulation experiments. Several examples are given of fitting instances of the new distribution to real and simulated data. It is noted that the new distribution is a member of the exponential family. Expressions for the gradient and Hessian of the log-likelihood of the new distribution are derived. The former facilitates the numerical maximization of the likelihood with optim(); the latter provides means of calculating or estimating the covariance matrix of of the parameter estimates. A discrepancy between estimates of the covariance matrix obtained by inverting the Hessian and those obtained by Monte Carlo methods is discussed.
\end{abstract}

\section{Introduction}

Modelling the distribution of discrete data sets can be problematic in that it is often the case that none of the "standard" distributions appears to be appropriate. It is possible to use a "completely nonparametric" approach (in other words, to apply multinomial distributions, specified in a very simple manner, by means of tables). However, this approach often turns out to be a little too flexible. In particular, in the context of hidden Markov models for discrete data (hmm.discnp, Turner 2020), the number of quantities to estimate rapidly becomes unwieldy. Estimates are unstable, the sensitivity of fitting algorithms to starting values is exacerbated, and problems with the convergence of fitting algorithms arise.

To address these problems, I developed a new discrete distribution, termed the " $\mathrm{db}$ " ("discretized Beta") distribution. The underlying idea is to define a family of distributions, for discrete data, with shape characteristics as flexible as those of the Beta family of continuous distributions. (See Johnson et al. 1995, Chapter 25, p. 210. See also the help for the dbeta() function in the stats package, R Core Team 2020, and Abramowitz and Stegun 1972, Chapter 6. The reader may also find it useful to access https://en.wikipedia.org/wiki/Beta_distribution.) The db distribution is closely related to the Beta distribution and has "shape" parameters, $\alpha$ and $\beta$, analogous to the shape parameters of the Beta distribution.

In addition to the shape parameters, the db distribution has two other parameters which specify the "support" of the distribution. These "support parameters" are not estimated from data but must be specified by the user prior to estimating the shape parameters. The support parameters are $n_{\text {top }}$ (a positive integer) and $\zeta$ (a logical scalar).

The parameter $n_{\text {top }}$ is the upper limit of the support of the specified distribution. If the parameter $\zeta$ is TRUE then zero origin indexing is to be used, in which case the support of the distribution is the set $\left\{0,1,2, \ldots, n_{\text {top }}\right\}$. Otherwise the support is $\left\{1,2, \ldots, n_{\text {top }}\right\}$. In the first case I use the notation $n_{\text {bot }}=0$ and in the second $n_{\text {bot }}=1$. The first form is convenient if the variable in question may be considered to be a count and zero counts are possible. Of course, one could structure the distribution always to have support of the form $\left\{0,1,2, \ldots, n_{\text {top }}\right\}$, simply by re-coding or shifting the data. However, in several of the examples with which I was concerned, it seemed more convenient to allow for a non-zero origin.

In some contexts the value of $n_{\text {top }}$ may be known (e.g., it may be analogous to the number of trials in a binomial experiment). In other contexts it must be chosen by the user, and the choice may be influenced by the observed values of the data. (See the section Choosing $n_{\text {top }}$.)

Like the Beta distribution upon which it is based, the db distribution is effectively unimodal. It can have two modes if they occur at the extremes of the support but otherwise can have only one. This characteristic is less than ideal, but seems to be unavoidable. It appears to be difficult to specify multimodal distributions (other than by way of mixtures, which are accompanied by other problems). Wikipedia (https://en.wikipedia.org/wiki/Multimodal_distribution, last accessed 30 March 2021) says "Bimodal distributions, despite their frequent occurrence in data sets, have only rarely been studied [citation needed] (sic). This may be because of the difficulties in estimating their parameters either with frequentist or Bayesian methods."

A referee of an earlier version of this paper suggested that the beta-binomial distribution be considered as an alternative to the new $\mathrm{db}$ distribution. This referee pointed out to me the paper "Modeling the patient mix for risk-adjusted CUSUM charts" by Philipp Wittenberg, which has interesting applications in medical science. In this paper, which is to appear in Statistical Methods in Medical 
Research, the beta-binomial distribution is used to model the distribution underlying a large data set of integer-based Parsonnet risk scores (see Parsonnet et al. 1989; see also Steiner et al. 2000). In addition to modeling the Parsonnet risk scores with the beta-binomial distribution, Wittenberg rescales these scores to lie between 0 and 1 and applies the Beta distribution.

The data in question are available (in a slightly modified form) in the spcadjust package (Gandy and Kvaloy 2013). I fitted both a db distribution and a beta-binomial distribution to these data and conducted a goodness of fit test in both instances. The tests indicate that neither distribution is actually appropriate. Both Monte Carlo $p$-values were 0.01. More detail is given in Example 6 in section Examples.

The beta-binomial distribution was introduced in Skellam (1948). Like the db distribution, it has flexibility of shape similar to that of the Beta distribution. However I have a couple of reservations about this distribution which I discuss in the following section.

\section{The beta-binomial distribution}

My first reservation about the beta-binomial distribution is that it is to a large extent focussed on dealing with data which appear to arise from binomial distributions but which are, in fact, "overdispersed". In other words, the focus is on data which exhibit extra-binomial variation. Such data have variance which is larger than it would be if the underlying distribution were indeed binomial.

The focus of the beta-binomial distribution on overdispersion would appear to make its application to underdispersed data problematic. Underdispersed pseudo-binomial data sets (i.e. data sets which have variance smaller than they would have if the underlying distribution were binomial) are rare, but they do exist. Examples are provided in the dbd package (see section Implementation). It is indeed possible to fit beta-binomial distributions to these examples, and goodness of fit tests indicate that the fits are adequate. However, the meaning of the resulting fits is questionable. The estimates of $s$ range from around 640 thousand to 78 million. Such large values of $s$ essentially indicate that there is no overdispersion, i.e., that the data are, in fact, from a binomial distribution. In other words, the estimates are trying as hard as they can to describe the true situation but cannot actually do so given the constraints of the model.

In fairness, it must be pointed out that the db distribution does not perform particularly well when applied to the data sets referred to above. There are no obvious theoretical problems with fitting the $\mathrm{db}$ distribution to underdispersed data. However, goodness of fit tests reject the adequacy of the fit of the $\mathrm{db}$ distribution to one of these data sets. In contrast, the beta-binomial distribution appears to fit this data set adequately. Further details are given in Example 7 in section Examples. The parameter estimates for the rejected fit of the db distribution appear to be excessively large, which might well raise suspicions. It is not clear how the values of parameter estimates obtained from the db distribution relate to under and overdispersion. This may be a topic to explore in future research.

My second reservation about the beta-binomial distribution is that (as revealed by fairly extensive simulation experiments) parameter estimation for this distribution can, from time to time, be unstable. The beta-binomial distribution may be conveniently parameterized in terms of a "success probability" $m$ (which must be strictly between 0 and 1) and an overdispersion parameter $s$ (which must be strictly positive). This is the parameterization chosen in the rmutil package (Swihart and Lindsey 2020). The reader may also find it informative to access https://en.wikipedia.org/wiki/Betabinomial_distribution, where the parameterization is expressed in terms of "shape" parameters $\alpha$ and $\beta$. These are related to $m$ and $s$ by $m=\alpha /(\alpha+\beta)$ and $s=\alpha+\beta$.

Moment estimators of the parameters of a beta-binomial distribution are explicitly available. However, these are often unsatisfactory in that the moment estimate of $s$ can turn out to be negative. Maximum likelihood estimates of the parameters may be obtained via numerical maximization (using, e.g., optim() from the stats package, automatically available in R). Starting values are, of course, required. If the moment estimates are outside of the required range, e.g., if $\hat{s}$ is negative, rough ad hoc starting values (e.g., $m=\epsilon$ or $m=1-\epsilon$, and $s=\epsilon$, where $\epsilon$ is equal to sqrt (.Machine\$double.eps)) appear to be adequate most of the time.

However, irrespective of starting values, the maximum likelihood estimate of $s$ is frequently far too large. In one instance, I simulated (using rbetabinom() from the rmutil package) 100 data sets, each with 30 observations, with $m=0.75, s=10$, and size (the number of trials) set equal to 10 . Maximum likelihood estimates of $s$, calculated using the true parameter values as starting values, ranged as high as 1038.82. (An " $h$ " plot of the estimates is shown in Figure 1.) The variance of these estimates was 13032.98. In contrast, the inverse of the Fisher information, calculated using the true parameter values and the data corresponding to the highest estimate of $s$ was 


$\begin{array}{rrr} & \mathrm{m} & \mathrm{s} \\ \mathrm{m} & 0.001121638 & 0.02524533 \\ \mathrm{~s} & 0.025245334 & 14.66327852\end{array}$

which indicates that the variance in question should be of the order of 15 .

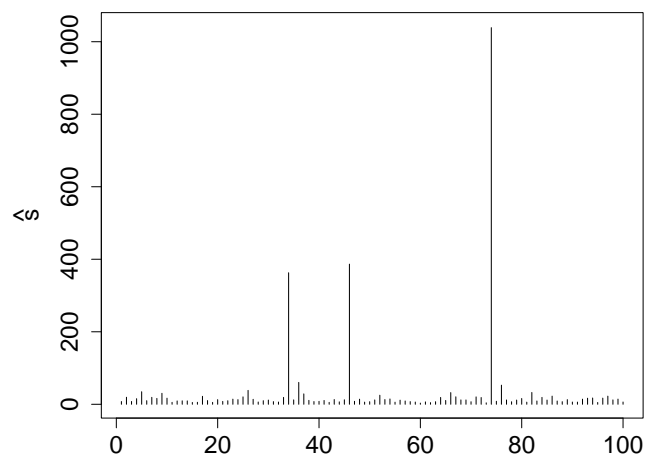

Figure 1: Estimates of the $s$ parameter of the beta-binomial distribution.

I encountered other problems - tendencies for errors to be thrown in various circumstances, including the application of optim() - in my exploration of the beta-binomial distribution, but there would appear to be no point in going into more detail here.

Despite these problems and my reservations about the beta-binomial distribution, I have included a complete set of tools for working with this distribution in the dbd package. These tools have a structure exactly analogous to that of the tools provided for working with the db distribution.

\section{Definition of the $\mathrm{db}$ distribution}

Conceptually, the probability mass function (PMF) of the db distribution is

$$
\begin{aligned}
\operatorname{Pr}\left(X=x \mid \alpha, \beta, n_{\mathrm{top}}, \zeta\right) & =\frac{1}{\kappa} f\left(\frac{x-n_{\text {bot }}+1}{n_{\text {top }}-n_{\text {bot }}+2}\right), \text { where } \\
\kappa & =\sum_{i=n_{\text {bot }}}^{n_{\text {top }}} f\left(\frac{i-n_{\text {bot }}+1}{n_{\text {top }}-n_{\text {bot }}+2}\right)
\end{aligned}
$$

$x=n_{\text {bot }}, n_{\text {bot }}+1, \ldots, n_{\text {top }}$. In (1), $f(\cdot)$ is the probability density function (pdf) of the Beta distribution with the first shape parameter equal to $\alpha$ and the second shape parameter equal to $\beta$. The probabilities given by (1) are the values of the corresponding Beta density, evaluated at equispaced points in the interior of the interval $(0,1)$, normalized to sum to 1 . However, it is possible and advantageous to express the definition of the $\mathrm{db}$ distribution in a direct manner without making reference to the Beta distribution.

Deriving the direct expression for the PMF of the db distribution from (1) is facilitated by noting that the Beta distribution is a member of the exponential family. From this, it follows that the $\mathrm{db}$ distribution as defined by (1) is, for fixed values of the support parameters $n_{\text {top }}$ and $\zeta$, also a member. An expression for the PMF of the db distribution, in exponential family form, is derived from the pdf of the Beta distribution in Appendix I.

From this derivation, it is seen that the PMF of the db distribution, given by (1), can also be expressed as

$$
\begin{aligned}
& \operatorname{Pr}\left(X=x \mid \alpha, \beta, n_{\mathrm{top}}, \zeta\right)=h(x) \exp \left\{\alpha T_{1}(x)+\beta T_{2}(x)-A(\alpha, \beta)\right\}, \\
& x=n_{\mathrm{bot}}, n_{\mathrm{bot}}+1, \ldots, n_{\mathrm{top}}, \text { where }
\end{aligned}
$$




$$
\begin{aligned}
h(x) & =\frac{\left(n_{\mathrm{top}}-n_{\mathrm{bot}}+2\right)^{2}}{\left(x-n_{\mathrm{bot}}+1\right)\left(n_{\mathrm{top}}-x+1\right)} \\
T_{1}(x) & =\log \left(\left(x-n_{\mathrm{bot}}+1\right) /\left(n_{\mathrm{top}}-n_{\mathrm{bot}}+2\right)\right) \\
T_{2}(x) & =\log \left(\left(n_{\mathrm{top}}-x+1\right) /\left(n_{\mathrm{top}}-n_{\mathrm{bot}}+2\right)\right. \text { and } \\
A(\alpha, \beta) & =\log \left(\sum_{i=n_{\mathrm{bot}}}^{n_{\mathrm{top}}} h(i) \exp \left\{\alpha T_{1}(i)+\beta T_{2}(i)\right\}\right) .
\end{aligned}
$$

Consequently the definition of the $\mathrm{db}$ distribution may be taken to be (2). This has the following advantage. The expression given by (2) is well-defined for all values of $\alpha$ and $\beta$ : positive, negative or zero, whereas (1) is well-defined only for $\alpha$ and $\beta$ strictly greater than zero. The difference is due to the fact that the pdf of the Beta distribution involves the expression $B(\alpha, \beta)=\Gamma(\alpha) \Gamma(\beta) / \Gamma(\alpha+\beta)$. This latter expression evaluates to $\infty$ (or Inf in R) if either $\alpha$ or $\beta$ is less than or equal to zero. Consequently, in such cases, (1) is undefined (being equal to Inf/Inf $=\mathrm{NaN}$ in R). Algebraically, $B(\alpha, \beta)$ cancels from (1) due to the division by $\kappa$, whence it does not appear in (2).

Although the $\mathrm{db}$ distribution is well-defined for negative parameter values, there are indications of problems in respect of such values. In practice, it may be advisable to restrict attention to positive values only. There certainly exist data sets - as can be demonstrated by simulation — for which the (maximum likelihood) estimates of the parameters are undeniably negative. Cursory experimentation using the dbd package indicates that in some instances, negative parameters are reasonably well estimated by maximum likelihood. E.g.,

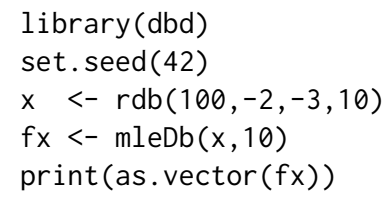

The preceding code produces estimates $\hat{\alpha}=-2.1645$ and $\hat{\beta}=-3.1365$. Ninety-five percent confidence intervals for $\alpha$ and $\beta$ (based on the variance entries of the "analytic" covariance matrix see section Implementation) are $[-3.0585,-1.2706]$ and $[-3.9443,-2.3297]$, which contain the true values, equal to -2 and -3 , respectively.

On the other hand, there are instances in which the maximum likelihood estimates are very large (either positive or negative) when the true values are relatively small and negative:

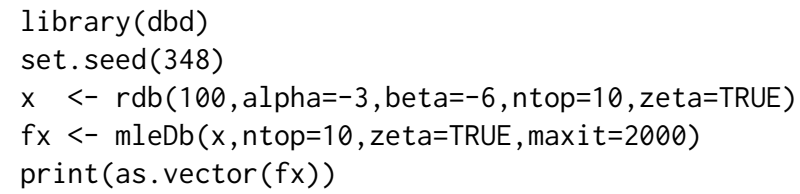

The resulting estimates are $\hat{\alpha}=73.18$ and $\hat{\beta}=-166.61$ which bear no relation to the "truth".

The only (as far as I can see) real advantage in the fact that the parameter values are permitted to be negative lies in the avoidance of various numerical issues that might otherwise arise in the estimation of parameters of a db distribution. In particular, the legitimacy of negative parameter values removes the necessity of imposing box constraints on the procedure for maximizing the likelihood. It also circumvents difficulties that can otherwise arise in evaluating the Hessian of the log-likelihood when one or both of the parameter estimates is close to zero.

Plots of the probability functions of a number of $\mathrm{db}$ distributions are shown in Figure 2. The shapes of these distributions mimic those of the corresponding Beta distributions.

\section{Choosing $n_{\text {top }}$}

In fitting a db distribution to an observed data set, it is often sensible to set $n_{\text {top }}$ equal to the maximum of the data. On the other hand, if there is a conceptual least upper bound for the support of the distribution (not found amongst the observed values), then one should set $n_{\text {top }}$ equal to this conceptual least upper bound. Finally, if the data are conceptually unbounded, then one might wish to set $n_{\text {top }}$ equal to the maximum of the observed data +1 . In this latter case, the value of $\operatorname{Pr}\left(X=n_{\text {top }}\right)$ might be interpreted as $\operatorname{Pr}\left(X \geq n_{\text {top }}\right)$. 

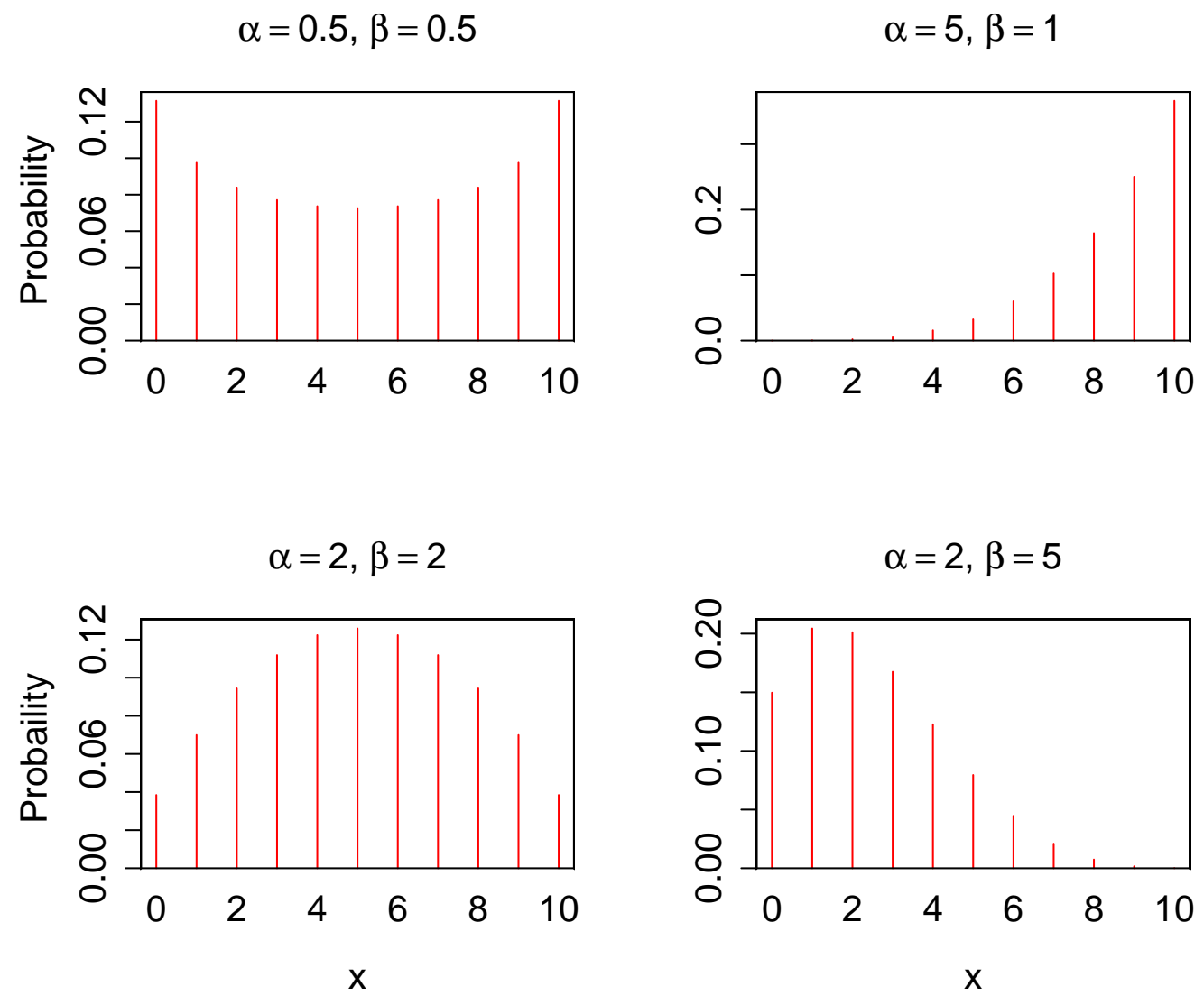

Figure 2: Probability mass functions of $\mathrm{db}$ distributions

\section{Implementation}

I have written an R package dbd (Turner 2021) to provide tools for working with the db distribution. The package supplies the four standard, " $\mathrm{d}$ ", " $\mathrm{p}$ ", " $\mathrm{q}$ " and " $\mathrm{r}$ " — density, probability, quantile and random number generation - functions, that are as a rule associated in $\mathrm{R}$ with any given distribution. In this setting, these functions are $\mathrm{ddb}(), \mathrm{pdb}(), \mathrm{qdb}()$, and $r \mathrm{db}()$. The package also contains functions $\operatorname{expValDb}()$ and $\operatorname{varDb}()$ to calculate the expected value (mean) and variance of a db distribution given a specification of its parameters. There is no closed-form algebraic expression for these quantities.

Another useful tool in the package is the function $\mathrm{mleDb}()$ which enables the easy estimation from data, of the parameters of the $\mathrm{db}$ distribution, via maximum likelihood. The function $\mathrm{mleDb}($ ) calls upon an undocumented function $\operatorname{meDb}()$ which calculates approximate moment estimates of the parameters to serve as starting values for the maximization of the likelihood. A user would not normally make direct use of meDb(). However, the approximation used by meDb() is of interest in its own right. This approximation is discussed in the section Estimation of parameters.

An alternative to $\mathrm{mleDb}()$ is the function exactMeDb(), which is also included in the dbd package. This function was suggested by a referee of an earlier version of this paper. It calculates "exact" moment estimates of the parameters by minimizing $(\bar{x}-\mu)^{2}+\left(s^{2}-\sigma^{2}\right)^{2}$ where $\bar{x}$ and $s^{2}$ are the sample mean and variance, respectively, and $\mu$ and $\sigma^{2}$ are the theoretical mean and variance. The latter two quantities are functions of $\alpha$ and $\beta$ and can be calculated from these parameters using expValDb() and $\operatorname{varDb}()$. The minimization is accomplished using optim(). "Theoretically", the minimum should be zero. The achieved minimum is provided as an attribute "minSqdiff" of the value returned by exactMeDb() so that the user can see how successful the minimization was. In section The quality of the estimates, the "exact" moment estimates are denoted by $\tilde{\alpha}$ and $\tilde{\beta}$.

Somewhat to my surprise, exactMeDb() performed (in the simulation experiments that I conducted) essentially as well as mleDb(). Occasionally exactMeDb() out-performed mleDdb() in terms of mean squared error. See section Quality of the estimators. On the other hand, it appears that exactmeDb() is substantially slower than $\mathrm{mleDb}()$. In a small simulation experiment I found that $\mathrm{mleDb}()$ was about 20 times as fast as exactMeDb() (with times measured as the "user" time returned by system. time()) 
when starting values were taken to be the approximate moment estimates, and nearly 30 times as fast when starting values were taken to be the true parameter values.

The function $\mathrm{mleDb}()$ returns an object of class "mleDb", and there is a corresponding plot () method to produce plots of the probability mass functions for distributions having parameters equal to the given estimates. There is also a stand-alone plotting function plotDb() which plots the PMF of a db distribution given specified parameters.

The package also has functions that provide means of estimating or calculating the covariance matrix of the parameter estimates. These functions enable the assessment of the uncertainty in the estimates of the parameters. The functions are: aHess() ("analytic Hessian"), nHess() ("numeric Hessian"), finfo(), and mcCovMat() (Monte Carlo-based estimate of the covariance matrix). The first three functions provide matrices whose inverses are estimates of (or in the case of finfo() equal to) the desired covariance matrix, given the supplied parameter values. The function nHess () calls upon optimHess() from the stats package. The function aHess() makes use of the expressions set out in Appendix II.

The values produced by aHess() and nHess() generally appear to be in very good agreement. However, if the parameter values are "unreasonably large" (e.g., $\alpha=150, \beta=400$ ), then there can be a substantial disparity between the values. In such instances, it would be inadvisable to trust either result.

Of course, the real reason for calculating the Hessian is to obtain an estimate of the covariance matrix of the parameter estimates. Another way to obtain an estimated covariance matrix is to use the Monte Carlo methods conveniently provided by the function mcCovMat () referred to above. Again, there is generally good agreement between the value produced by mcCovMat () and the inverse of the Hessian produced, e.g., by aHess(). However, unless the sample size is quite large, the variance entries of the inverse Hessian are noticeably smaller than those of the matrix returned by mcCovMat ():

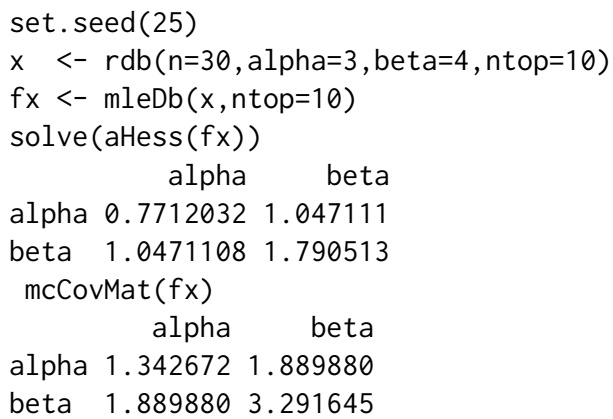

Further discussion of this phenomenon is to be found in Appendix III.

The dbd package also contains a function 1lPlot() for plotting log-likelihood surfaces and a function gof () for performing tests of goodness of fit for the db distribution. The llPlot() function may be useful in diagnosing problems with parameter estimation should these arise. The tests effected by gof() may be either chi-squared-based tests or Monte Carlo tests. Users should be aware that Monte Carlo tests (which use a relatively small number of simulations) are random. Performing a Monte Carlo test is not the same as simulating a large number of test statistics (under the null hypothesis) in order to approximate the null distribution of the statistic. See, for example, Baddeley et al. (2015, Section 10.6, p. 384) for some discussion of such tests.

\section{Estimation of parameters}

There is, unsurprisingly, no closed-form for any sort of estimates of the shape parameters of a db distribution. Estimates may, however, be calculated reasonably easily via (numerical) maximum likelihood or by solving for moment estimates numerically. The functions mleDb() and exactMeDb() from the dbd package, discussed in the section Implementation make use of the optim() function from the stats package (automatically available in $\mathrm{R}$ ) to effect the calculations.

The optim() function requires starting values for the parameters being estimated. It turns out that adequate starting values can be produced, as indicated in the section Implementation, via a (very!) rough explicit approximation to the method of moments. To develop the approximation, it is necessary to go back to the conceptual definition of the $\mathrm{db}$ distribution expressed in terms of the Beta distribution (1). In terms of the conceptual definition, the mean and variance of a db distribution with 
shape parameters $\alpha$ and $\beta$ may be written as

$$
\begin{aligned}
\mu & =\frac{1}{\kappa} \sum_{i=0}^{n} i f((i+1) /(n+1)) \\
\sigma^{2} & =\frac{1}{\kappa} \sum_{i=0}^{n}(i-\mu)^{2} f((i+1) /(n+1)),
\end{aligned}
$$

where $f(\cdot)$ is the probability density function of the Beta distribution with shape parameters $\alpha$ and $\beta$, $n$ is equal to $n_{\text {top }}$ (the maximum value of the support of the distribution), and $\kappa$ is the normalizing constant

$$
\kappa=\sum_{i=0}^{n} f((i+1) /(n+1)) .
$$

In the foregoing, zero origin indexing (i.e., that $\zeta$ is TRUE) is assumed. This is of no real consequence given that the method is so rough to start with.

To get approximate expressions for the mean and variance of the distribution, one may manipulate the sums in the expressions for $\mu, \sigma^{2}$, and $\kappa$ into the form of Riemann sums that approximate integrals. This reveals that

$$
\mu \approx \frac{(n+2)^{2}}{\kappa} \int_{0}^{1} x f(x) d x-1
$$

and that

$$
\kappa \approx(n+2) \int_{0}^{1} f(x) d x .
$$

The integral of $x f(x)$ is the mean of the associated Beta distribution, $\alpha /(\alpha+\beta)$ (Johnson et al. 1995, Chapter 25, equation 25.15a) and the integral of $f(x)$ is, of course, just 1 , whence $\kappa \approx n+2$. Therefore,

$$
\mu \approx \frac{(n+2) \alpha}{\alpha+\beta}-1
$$

Proceeding similarly, one finds that

$$
\sigma^{2} \approx \frac{(n+2)^{2} \alpha \beta}{(\alpha+\beta)^{2}(\alpha+\beta+1)}
$$

This latter result makes use of the fact that the variance of the associated Beta distribution is

$$
\frac{\alpha \beta}{(\alpha+\beta)^{2}(\alpha+\beta+1)}
$$

(Johnson et al. 1995, Chapter 25, equation 25.15b).

To calculate the approximate method of moments estimates, which I shall denote by $\breve{\alpha}$ and $\breve{\beta}$ respectively, equate the foregoing approximate expressions for $\mu$ and $\sigma^{2}$ to the observed sample mean and variance $\left(\bar{x}\right.$ and $\left.s^{2}\right)$ and solve for $\alpha$ and $\beta$. One obtains

$$
\begin{aligned}
\breve{\alpha} & =\frac{(n+2)^{2} a}{s^{2}(a+1)^{3}}-\frac{1}{a+1} \\
\breve{\beta} & =a \breve{\alpha},
\end{aligned}
$$

where for convenience, I have set $a=(n+1-\bar{x}) /(1+\bar{x})$.

Note that although the Beta distribution is undefined for non-positive values of $\alpha$ and $\beta$, the foregoing approximate moment estimation procedure can be applied without problem to data for which non-positive parameter estimates are appropriate.

It must be emphasized here that the explicit moment estimation procedure discussed above is not intended to be applied by users. It is provided for the purpose of producing starting values for the maximum likelihood and "exact" moment estimation procedures. The estimates produced via the explicit moment estimation procedure are not very good, and in general, appear to be substantially biased. (See Figure 4.) Despite this, they seem to be adequate as starting values.

\section{Quality of the estimators}

I investigated the question of how well the estimation procedures perform by means of a number of simulation experiments. 


\section{Some confidence intervals for the parameters}

In the first experiment, I determined interval estimates of $\alpha$ and $\beta$ for a small grid of true values of these parameters. One hundred samples were generated for each combination of the true parameter values, and the means and standard errors of these estimates were calculated. Each sample was of size of 100 . For each combination of the true parameters, $95 \%$ confidence intervals (mean $\pm 1.96 \times$ standard error) for the individual parameters were then calculated. Table 1 displays the sample means of the estimates of $\alpha$ and $\beta$ and the corresponding confidence intervals.

Half of the 18 confidence intervals failed to cover the true values. For the $(3,3)$ combination, the $\beta$ interval failed to cover. For the $(3,6),(6,6),(9,3)$, and $(9,9)$ combinations, both intervals failed to cover. Where the confidence intervals failed to cover, they missed on the high side. As shall be seen later on, (Figure 6 in subsection Asymptotic bias in the maximum likelihood estimates), this would seem to be the expected behavior. The amounts by which the confidence intervals missed the true values were not egregiously large. The worst case was for the $(9,3)$ combination, where the lower endpoint of the confidence interval for $\alpha$ was greater than 9 by 0.294 .

\begin{tabular}{|cc|c|c|c|c|}
\hline$\alpha$ & $\beta$ & $\overline{\hat{\alpha}}$ & $95 \%$ CI for $\alpha$ & $\overline{\hat{\beta}}$ & $95 \%$ CI for $\beta$ \\
\hline \hline 3 & 3 & 3.014 & $(3.005,3.023)$ & 3.011 & $(3.003,3.019)$ \\
3 & 6 & 3.029 & $(3.021,3.038)$ & 6.058 & $(6.041,6.075)$ \\
3 & 9 & 3.018 & $(3.01,3.026)$ & 9.056 & $(9.031,9.08)$ \\
6 & 3 & 6.056 & $(6.04,6.072)$ & 3.015 & $(3.007,3.022)$ \\
6 & 6 & 6.029 & $(6.012,6.046)$ & 6.042 & $(6.025,6.059)$ \\
6 & 9 & 6.019 & $(6.004,6.035)$ & 9.022 & $(8.999,9.045)$ \\
9 & 3 & 9.002 & $(8.974,9.029)$ & 3.008 & $(2.998,3.017)$ \\
9 & 6 & 9.032 & $(9.007,9.057)$ & 6.014 & $(5.997,6.031)$ \\
9 & 9 & 9.032 & $(9.006,9.057)$ & 9.005 & $(8.979,9.031)$ \\
\hline
\end{tabular}

Table 1: Some sample mean parameter estimates and $95 \%$ confidence intervals for the true values.

\section{Comparison of estimators using mean squared error}

I next conducted an experiment to investigate how well the two sorts of moment estimator compared with the maximum likelihood estimator. In this experiment, 100 samples, each of size 100, were generated (using $\mathrm{rdb}(\mathrm{)})$ ) from distributions

$$
\mathrm{db}\left(\alpha_{i}, \beta_{j}, n_{\text {top }}=10, \zeta=\text { TRUE }\right),
$$

with $\alpha_{i}$ and $\beta_{j}$ varying over the set $\{0,1,2, \ldots, 9,10\}$. For each of the three possible estimators (explicit but approximate method of moments, "exact" method of moments, and maximum likelihood), the mean squared error of the estimates, corresponding to the appropriate set of 100 samples, was calculated. The mean square error is defined as

$$
\operatorname{MSE}=(\alpha-\bar{\alpha})^{2}+(\beta-\bar{\beta})^{2}+s_{\alpha}^{2}+s_{\beta}^{2} .
$$

In the foregoing, $\bar{\alpha}$ and $s_{\alpha}^{2}$ are the sample mean and variance of the 100 values of the estimates of $\alpha$ $\left(\breve{\alpha}_{i}, \tilde{\alpha}_{i}, \hat{\alpha}_{i}\right.$ as the case may be) arising from the 100 samples generated for the given pair of parameter values. Similarly for $\bar{\beta}$ and $s_{\beta}^{2}$. A subset of the MSE values that were produced is presented in table 2.

It is worth mentioning that the MSEs of the "exact" moment estimates and of the maximum likelihood estimates were not adversely affected by the possibly poor starting values provided by the approximate moment estimates. In a simulation experiment, it is possible to use the true values of the parameters as starting values. Doing so gave rise to estimates that were virtually identical to those obtained when the approximate moment estimates were used to start the optimization.

A plot of the "exact" moment estimates against the maximum likelihood estimates is shown in Figure 3. The MSE for the "exact" moment estimates tracked the MSE for the maximum likelihood estimates almost exactly except for one striking outlier, corresponding to the parameter pair $\alpha=10$, $\beta=0$. A little bit of further experimentation indicated that this outlier is a one-off aberration, and that both "exact" moment estimation and maximum likelihood estimation are subject to occasional instability. More simulation experiments could be considered, but there are too many possibilities for this line of enquiry to be pursued in the current paper.

Unsurprisingly, the MSE of estimates produced by the approximate moment method did not track that of the maximum likelihood estimates nearly as closely, as shown in Figure 4. There is some 


\begin{tabular}{|r|r|rrr|}
\hline \multicolumn{2}{|c|}{} & \multicolumn{3}{|c|}{ Mean squared error } \\
\hline$\alpha$ & $\beta$ & Approx. Mom. & Exact Mom. & Max. Like. \\
\hline 0 & 0 & 1.24 & 0.10 & 0.10 \\
5 & 0 & 55.58 & 8.13 & 8.03 \\
10 & 0 & 636.59 & 102.20 & 45.55 \\
0 & 2 & 4.93 & 0.69 & 0.71 \\
5 & 2 & 1.10 & 1.10 & 1.09 \\
10 & 2 & 9.24 & 7.11 & 6.83 \\
0 & 4 & 27.60 & 2.50 & 2.43 \\
5 & 4 & 1.02 & 1.02 & 1.06 \\
10 & 4 & 3.19 & 3.28 & 3.45 \\
0 & 6 & 94.64 & 8.58 & 8.53 \\
5 & 6 & 1.34 & 1.34 & 1.37 \\
10 & 6 & 3.08 & 3.06 & 3.13 \\
0 & 8 & 292.97 & 28.21 & 28.08 \\
5 & 8 & 1.48 & 1.47 & 1.45 \\
10 & 8 & 3.53 & 3.53 & 3.69 \\
0 & 10 & 556.54 & 50.80 & 52.78 \\
5 & 10 & 3.82 & 3.80 & 3.93 \\
10 & 10 & 5.40 & 5.40 & 5.78 \\
\hline
\end{tabular}

Table 2: Subset of the MSE values plotted in Figures 3, 4 and 5.

suggestion that the MSE pairs are close to the " $y=x$ " line for small values of MSE, but for values larger than about five, the plot of the MSE values becomes rather wild.

Evidence from the simulation experiments described here indicates that the MSE becomes large when the difference between $\alpha$ and $\beta$ becomes large. Figure 5 displays a plot of the maximum likelihood MSE against $|\alpha-\beta|$. This figure indicates that the MSE values are all of roughly the same "moderate" size until the absolute difference becomes greater than or equal to six. At this point the values start to increase substantially.

\section{Asymptotic bias in the maximum likelihood estimates}

The squared bias component of the MSE for the maximum likelihood estimates was substantial. However, the simulation experiment described above used a sample size of 100 exclusively. To assess the impact of sample size on the bias in the estimates, I undertook a further simulation experiment in which I set $\alpha$ and $\beta$ to have true values 0 and 10 respectively, which were the values that led, in the foregoing experiment, to the largest MSE. The sample size was allowed to range over the set $\{100,200,300,500,1000,5000\}$. Five hundred simulated samples were generated in each instance, and the means and standard errors of the bias in parameter estimates were calculated.

Ninety-five percent confidence intervals for the mean bias at were plotted for both the $\alpha$ and $\beta$ estimates. These plots are shown in Figure 6. This figure indicates that for this pair of true parameter values, the bias remains "significantly" different from 0 until the sample size reaches the surprisingly large value of 2000 .

\section{Examples}

\section{Example 1: Simulated binomial data}

For an initial example, I simulated 100 data from a binomial distribution $\operatorname{Bin}(10,0.25)$ and fitted a db distribution to that. I set $\zeta=$ TRUE and $n_{\text {top }}=10$ (the "conceptual" upper bound). A plot of the resulting fit is shown in Figure 7 . The fit is consistent with the other possible values of the probabilities of the various counts.

\section{Example 2: The Downloads data}

The Downloads data from Weiß (2018) consist of a time series (of length 267) of the observed daily number of downloads of a $\mathrm{T}_{\mathrm{E}} \mathrm{X}$ editor for the period from June 2006 to February 2007. These data are 


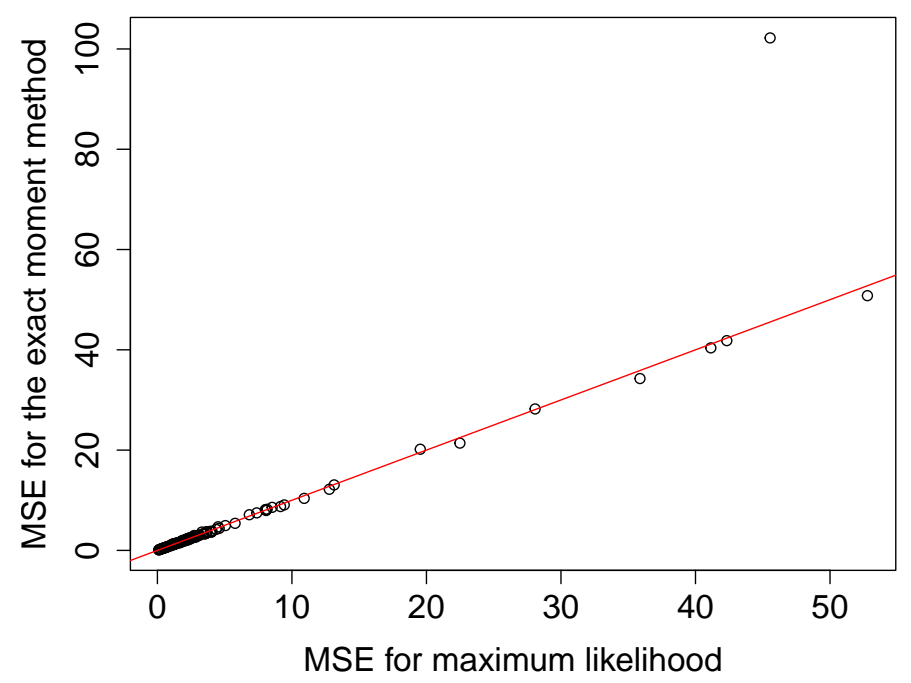

Figure 3: The MSE from "exact" moment estimation plotted against the MSE from maximum likelihood estimation. The superimposed red line is the line of "exact agreement" between the two estimators, i.e. it has slope 1 and intercept 0 .

available as Downloads in the CRAN package hmm.discnp. They can also be obtained from the Wiley website https://www. wiley. com/en-gb/An+Introduction+to+Discrete+Valued+Time+Series-p9781119096962, by clicking on "Downloads" and then on the "Download" button next to "Datasets". This will provide a zip archive of all of the data sets from Weiß's book.

Prima facie, it might seem plausible that these data are Poisson-distributed, but they are, in fact, much too overdispersed to be Poisson; the sample mean is 2.401, whereas the sample variance is 7.506 . Weiß finds that an INAR(1) (integer-valued autoregressive, $p=1$ ) model provides a good fit. In fitting a db distribution to these data, I took $\zeta=$ TRUE (zero counts are observed) and ntop $=15$ ( $1+$ the observed maximum of the data, which is 14). This db fit yields a mean of 2.451 and a variance of 7.461. A plot of this fit is shown in Figure 8. Of course, simply fitting a db distribution is not really appropriate since this treats the data as being i.i.d., and as Weiß's analysis shows, there is strong evidence of serial dependence in these data. Moreover, a goodness of fit test yields a $p$-value of 0.03 (see the help for gof ( ) in the dbd package). One would thereby reject the hypothesis that the fit of the $\mathrm{db}$ distribution is adequate at the 0.05 significance level.

In other analyses of these data, the details of which it is inexpedient to discuss here, I have fitted hidden Markov models with marginal db distributions and varying numbers of states to these data. I used both AIC and BIC to select the number of states. Both criteria indicate that a two-state model is optimal, i.e., a model involving serial dependence is chosen over the model in which the data are i.i.d.

\section{Example 3: The Sydney Coliform Count data}

These data were analyzed in Turner et al. (1998). In that paper, the data were modeled after a certain transformation had been applied, as having a hidden Markov model structure with marginal Poisson distributions. I have since discretized these data into five (ordered) categories. The resulting data set is available as SycColDisc in the package hmm.discnp. I fitted db distributions to subsets of these data, treating them as having the numeric values $1,2, \ldots, 5$, taking $n_{\text {top }}=5$ and $\zeta=$ FALSE.

Plots of the fits, together with the observed proportions, are shown in Figure 9, for the Bondi East data at each of the four depths, 0, 20, 40, and 60 meters.

\section{Example 4: The Sydney Coliform Count data (continued)}

In general, it may be of interest to provide graphical representations of the uncertainty in the estimates of the parameters of $\mathrm{db}$ distributions. This can be done by plotting (say) $95 \%$ confidence ellipses around the point estimates. The ellipse package (Murdoch and Chow 2018) provides convenient means of plotting such ellipses.

In the context of the current example, it is also of interest to examine whether there are differences amongst the distributions associated with the various depth and location combinations. Such exami- 


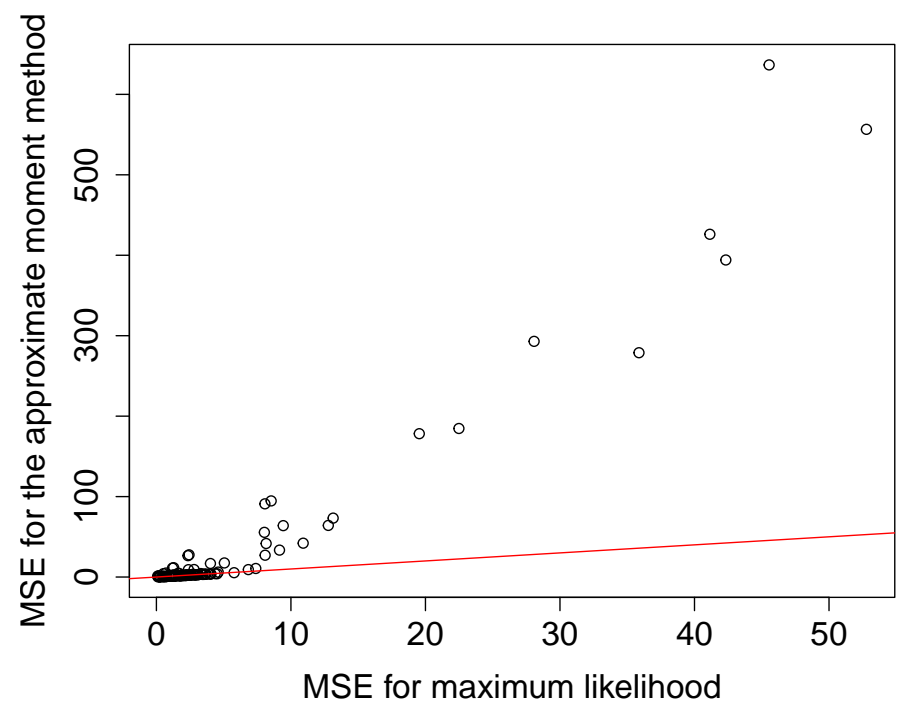

Figure 4: The MSE from approximate moment estimation plotted against the MSE from maximum likelihood estimation. The superimposed red line is the line of "exact agreement" between the two estimators, i.e. it has slope 1 and intercept 0 .

nation can also be effected by means of plotting confidence ellipses. In this setting, one would plot confidence ellipses for the differences between the parameters corresponding to different combinations. Assuming that the samples are independent (possibly problematic here), the covariance matrix on which to base the confidence ellipse for the difference between the parameters is the sum of the two individual covariance matrices.

It is also possible to test the hypothesis that the distributions corresponding to a number of different combinations of depths and locations are all identical (again assuming the samples to be independent) by means of a likelihood ratio test. The foregoing ideas can be illustrated using the four different depths at the "BondiE" location. Confidence ellipses for the parameters corresponding to the four depths are shown in Figure 10. Confidence ellipses for the six pairwise differences are shown in Figure 11. Code for effecting the likelihood ratio test is as follows:

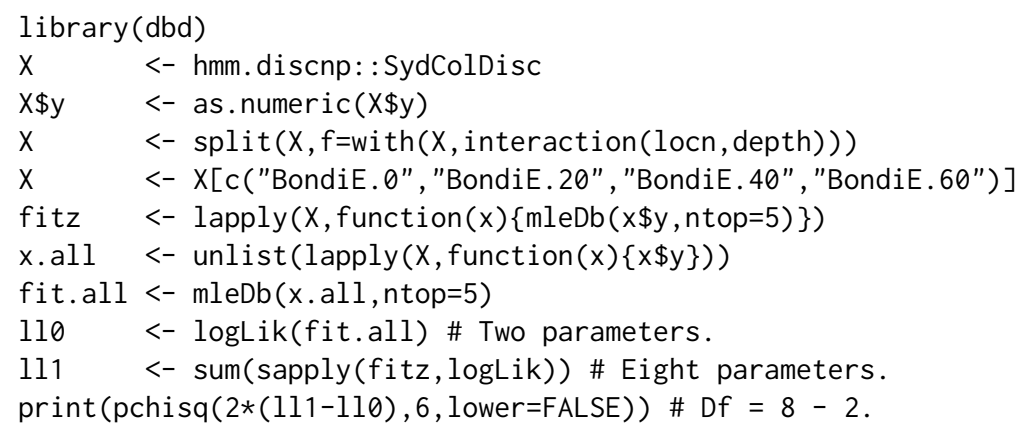

The resulting $p$-value is 0.9781 ; i.e., there is no evidence at all of any differences. This conclusion is confirmed by Figure 11, wherein it can be seen that the $95 \%$ confidence ellipses all contain the point $(0,0)$. It is also in accordance with the visual impression given by Figure 9 in which the plots of the four distributions all look very similar.

An analogous exercise was done involving measurements all made at a depth of 60 meters, at four of the seven locations (Longreef, Bondi East, Malabar Offshore, and North Head Offshore; two "controls" and two "outfalls"). The likelihood ratio test yielded a $p$-value equal to $6.37 \times 10^{-9}$, i.e., effectively zero. The origin $(0,0)$ was exterior to the $95 \%$ confidence ellipses for the pairwise differences in four of the six instances.

Note that the foregoing analyses of the Sydney Coliform data are superficial in that they take no account of the serial dependence of these data. Undertaking analyses that accommodate serial dependence would lead us much too far afield. 


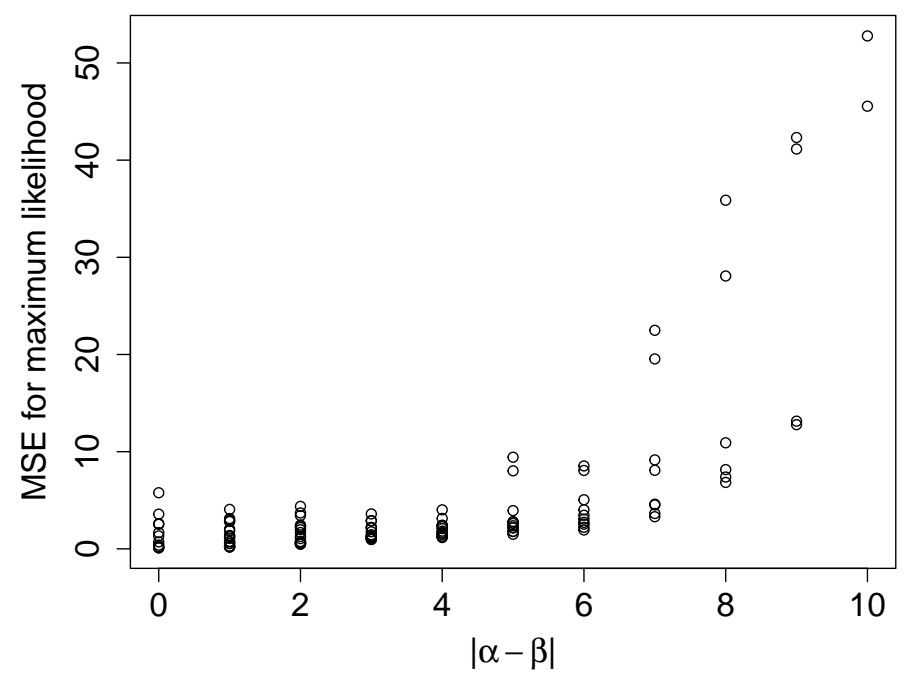

Figure 5: The MSE from maximum likelihood estimation plotted against the absolute value of the difference the parameters in the corresponding parameter pair.

\section{Example 5: Monocyte counts and psychosis ratings}

The monocyte counts and psychosis ratings data arose in a study initiated by Jonathan Williams, who was at the start of the study, working for the Northland District Health Board in New Zealand. The study is still ongoing, and the results have not yet been published. The data involved cannot be publicly released due to patient confidentiality issues.

This example is really what motivated the development of the db distribution. The data consist of pairs of sequences of observations of monocyte blood counts, discretized to a 1 to 5 scale, and ratings of severity of psychosis on a 0 to 4 scale. The observations were made on 1258 patients. They were made at irregularly spaced times, and there were varying numbers of observations per patient. The different types of sequence were not observed at the same times, and there were usually different numbers of observation between the types.

The analysis of these data was intricate, and the details cannot be gone into here. The crucial feature of the analysis is that hidden Markov models, whose marginal distributions were $\mathrm{db}$ distributions were fitted to both types of sequence. The value of $n_{\text {top }}$ was taken to be 5 for the monocyte counts and 4 for the psychosis ratings, and that of $\zeta$ to be FALSE for the monocyte counts and TRUE for the psychosis ratings.

For each type, a three-state model was chosen (by means of a cross-validation technique). Plots of the $\mathrm{db}$ distributions corresponding to each of the three states are shown for the monocyte counts in Figure 12 and for the psychosis ratings in Figure 13.

\section{Example 6: The Parsonnet scores from the cardiacsurgery data}

As discussed in the introduction, I fitted both a $\mathrm{db}$ and a beta-binomial distribution to these data and conducted goodness of fit tests. In the case of the $\mathrm{db}$ distribution, I chose $n$ top $=71$ and set zeta $=$ TRUE (since zeroes appear in the data). In the case of the beta-binomial distribution, I set size $=71$. The value 71 is that which was used in the paper by Wittenberg (to appear in Statistical Methods in Medical Research) referred to on page 485. 

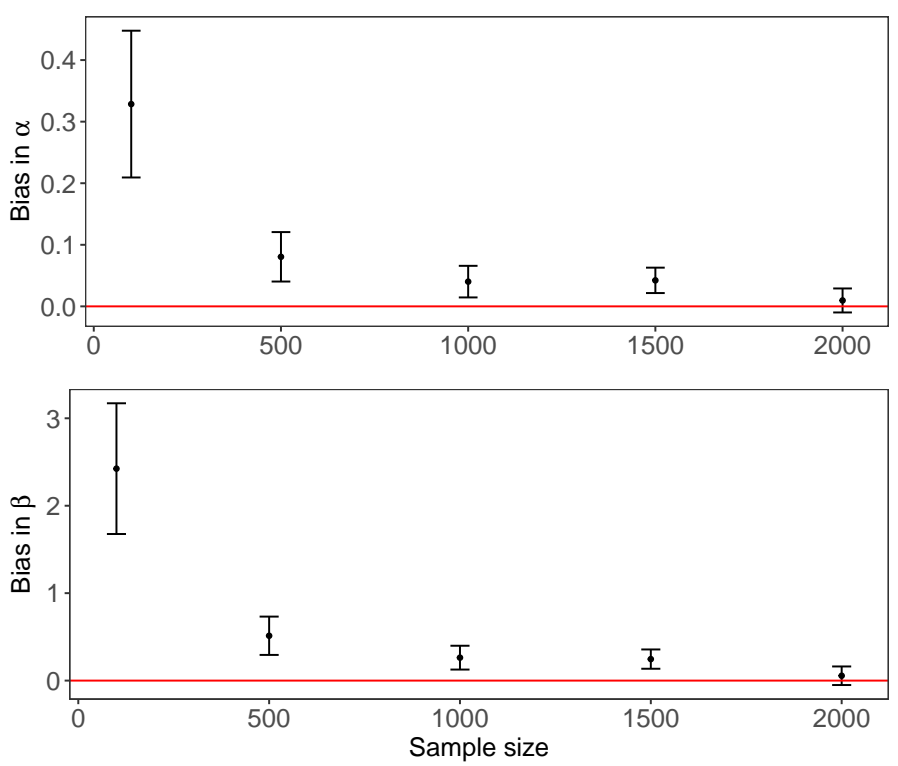

Figure 6: Ninety-five percent confidence intervals for the mean bias in estimates of $\alpha=0$ and $\beta=10$, plotted against the sample size. Five hundred sample were generated for each sample size. The red horizontal lines are the desired zero bias level.

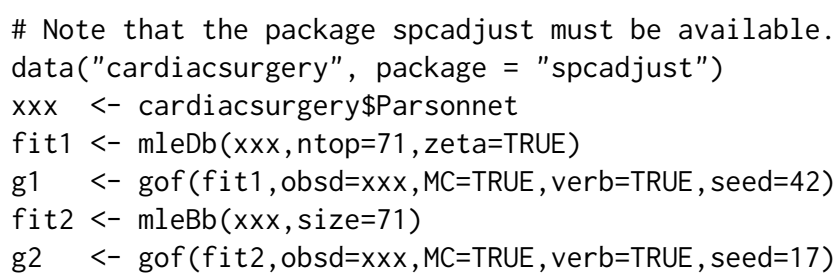

Seeds were set in the calls to gof ( ) in order for the Monte Carlo $p$-values to be reproducible. In both cases, the value of nsim was left at its default value of 99, which yielded a Monte Carlo $p$-value of 0.01 . The values of the test statistic were huge, 12792.5 in the case of the db distribution, and 4004.668 in the case of the beta-binomial distribution. I therefore strongly suspect that if nsim had been increased to, say 9999, then the $p$-values would have been 0.0001 . Howeveri, I have not checked this out.

Plots of the fits (not shown) can be produced by:

plot (fit1, obsd=xxx, main="db")

plot (fit2, obsd=xxx, main="beta-binomial)

These plots reveal that there are indeed substantial discrepancies between the fitted probabilities and the observed proportions. The fitted probabilities from the dbd distribution, although differing significantly from the observed proportions, are "not too different" from each other. This can be seen from the plot (again not shown) that can be produced by:

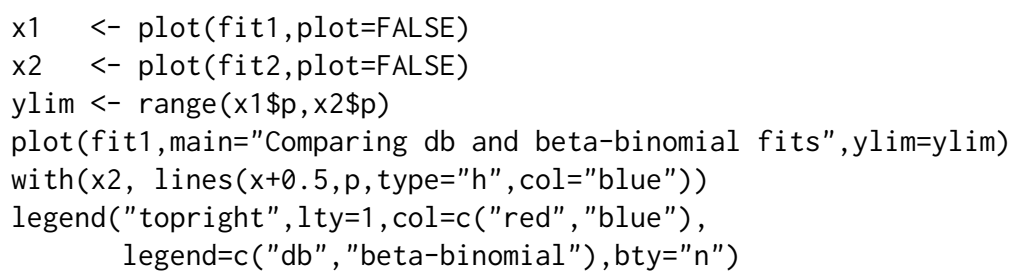

The large size (5595), of the data set that is involved here, revealed an interesting timing issue. The goodness of fit test took a great deal (of the order of 50 times) longer for the beta-binomial distribution than for the $\mathrm{db}$ distribution. Some rudimentary timing experiments revealed that for data sets of this size, the random number generator rbetabinom() from the rmutil package is about 50 times slower than $\mathrm{rdb}($ ) from the dbd package. I have not investigated the reason for this phenomenon. 


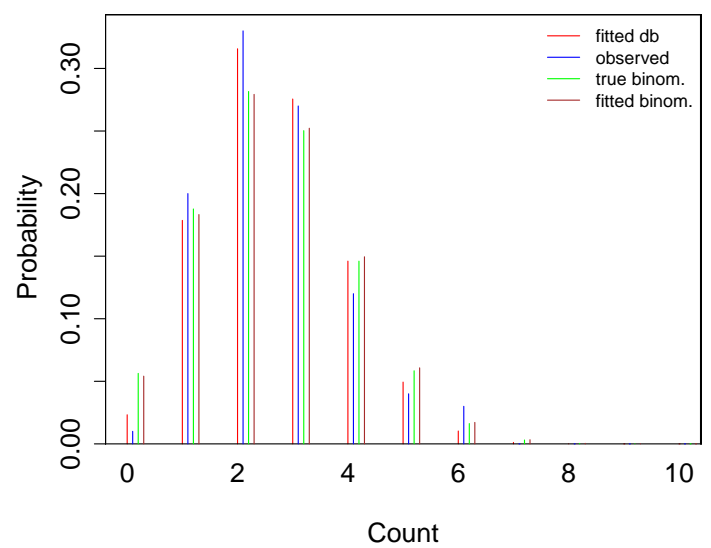

Figure 7: Fit of a db distribution to a sample from a $\operatorname{Bin}(10,0.25)$ distribution. Also shown are the observed proportions, the true binomial probabilities, and the fitted binomial probabilities.

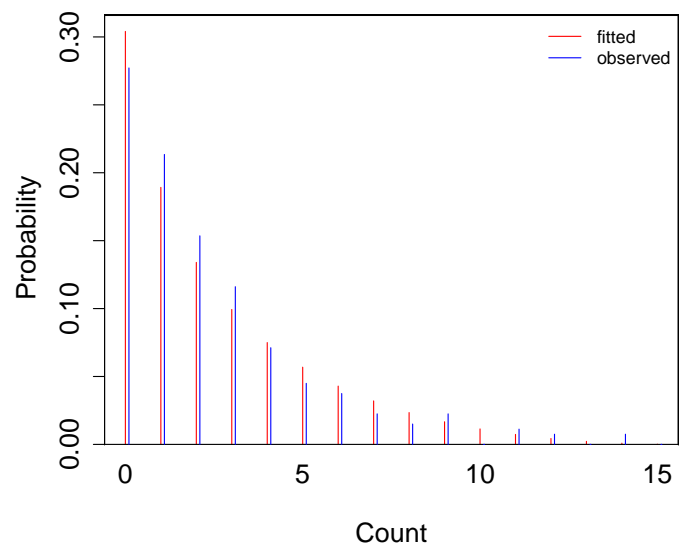

Figure 8: Fit of a db distribution to the Downloads data from Weiß (2018). Also shown are the observed proportions.

\section{Example 7: Underdispersed data}

As mentioned in the introduction, the dbd package provides two data sets which are of the nature of binomial data but appear to be underdispersed relative to the binomial distribution. Here are some examples which illustrate fitting $\mathrm{db}$ and beta-binomial distributions to these data.

The horse race prediction data from the dbd package (see the help for hrsRcePred) provides four examples.

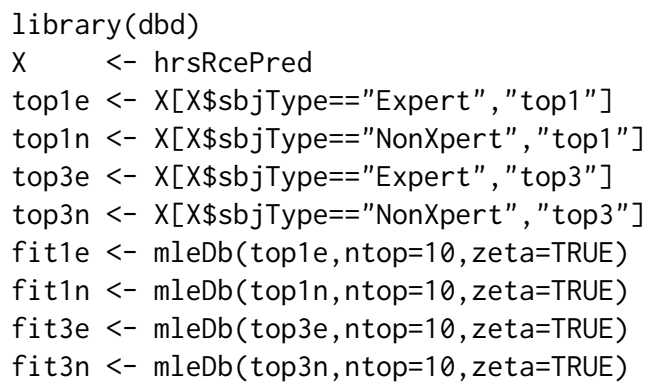

The ratios of the raw variance to the putative binomial distribution variance are $0.4895,0.4100,0.5718$ 

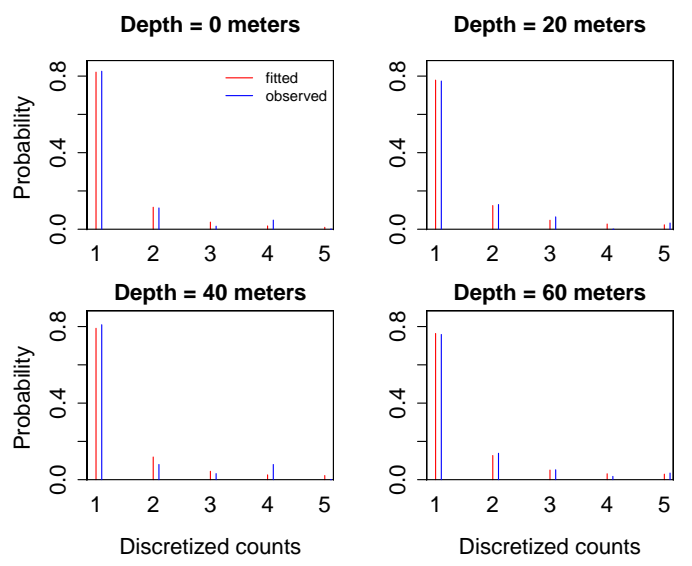

Figure 9: Fits of $\mathrm{db}$ distributions to discretized Sydney coliform count data from the Bondi East location, at depths equal to $0,20,40$, and 60 meters. Also shown are the observed proportions.
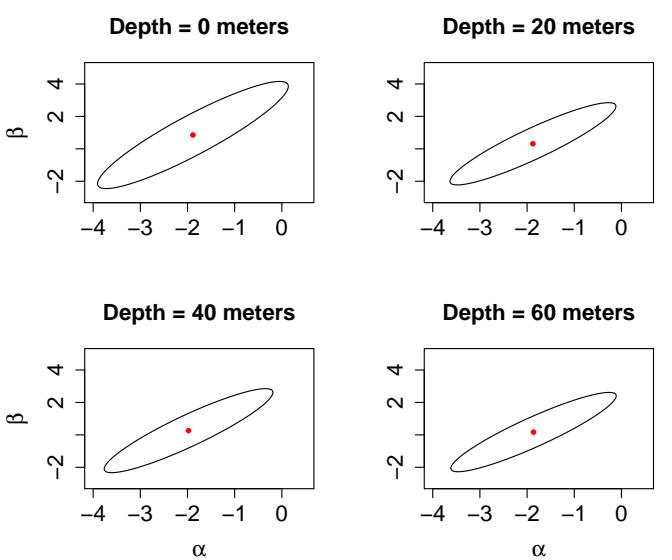

Figure 10: Ninety-five percent confidence ellipses for the parameters of the db distributions fitted to the data from the Bondi East location at depths 0, 20, 40, and 60 meters.

and 0.7745 respectively. All ar substantialy less than 1 , whence these data sets appear to be indeed underdispersed.

The fitting procedures proceeded without complaint for all four data sets. Goodness of fit tests (two of which required increasing maxit from its default value) indicate adequate fit for three of the four data sets.

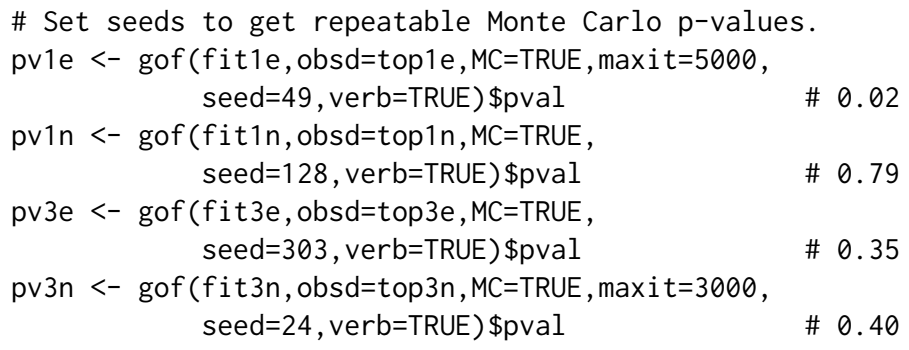

There is significant evidence that the $\mathrm{db}$ distribution is not appropriate for the top $1 \mathrm{e}$ data ( $p$-value $=0.02$ ). For the other three data sets, the $p$-values are all large, indicating that there is no evidence of any problems with any of these fits. 

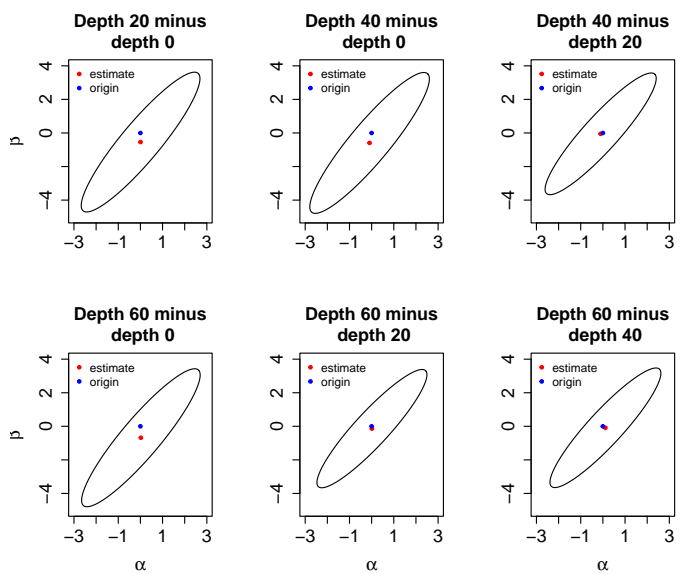

Figure 11: Ninety-five percent confidence ellipses for pairwise differences between the parameter vectors of the $\mathrm{db}$ distributions fitted to the data from the Bondi East location at depths 0, 20, 40, and 60 meters.

Note that for the problematic fit (i.e., fit1e), the parameter estimates might be considered to be excessively large:

$$
\begin{array}{rr}
\text { alpha } & \text { beta } \\
145.14659 & 21.23249
\end{array}
$$

This may be indicative of problems.

The beta-binomial distribution fits acceptably to all four of the data sets top1e, top $1 \mathrm{n}$, top3e, and top3n. For example, for the first of these data sets (for which the fit of the db distribution was rejected), the following code produces a Monte Carlo $p$-value of 0.11 :

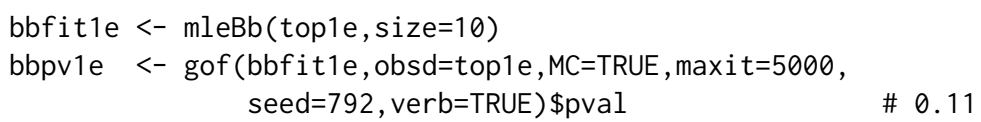

(For the other three data sets, Monte Carlo $p$-values of $0.64,0.62$, and 0.75 were obtained.)

Another two examples of underdispersed data are provided by the visual recognition data from the dbd package (see the help for visRecog). The ratios of the raw variance to the putative binomial distribution variance for these data are 0.7635 and 0.7979 . The Monte Carlo $p$-values from fitting the $\mathrm{db}$ distribution were 0.92 and 0.71 , and those from fitting the beta-binomial distribution were 0.97 and 0.83 .

As a "reality check", it is worth noting that fitting a simple binomial distribution to these data sets yielded Monte Carlo $p$ values, from goodness of fit tests, equal to $0.13,0.61,0.72$, and 0.70 for the hrsRcePred data, and 0.98 and 0.81 for the visRecog data. That is, binomial distributions fit these data sets acceptably despite their apparent underdispersion.

\section{Concluding remarks}

The $\mathrm{db}$ distribution is a new distribution that can be applied to any sort of data that takes values in a finite discrete set. It is an ad hoc distribution and does not require any theoretical justification in terms of properties that the data may have. It is very flexible, with the restriction that (as remarked in the Introduction) it is effectively unimodal. The values of the distribution are integers varying from 0 to $n$ or from 1 to $n$ for some $n$, and data to which a db distribution is to be fitted must be converted (recoded) into that form. In order that the fit should make practical sense, the data should, generally speaking, bear some relation to counts or at least be ordered. However, there is no theoretical requirement that this should be the case.

A number of examples have been given in this paper illustrating the fit of the $\mathrm{db}$ distribution to different data sets. These examples show that the db distribution may reasonably be expected to be useful to data analysts who need to deal with discrete data that do not conform to one of the standard distributions. 
State 1

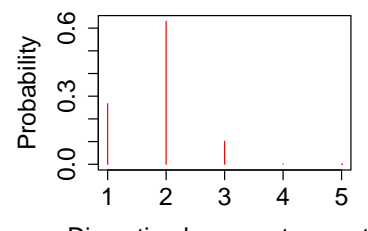

State 3

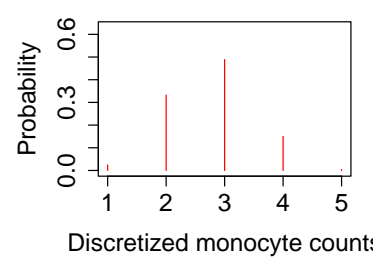

State 2

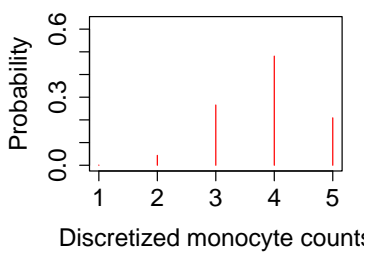

model fitted to the monocyte count data.
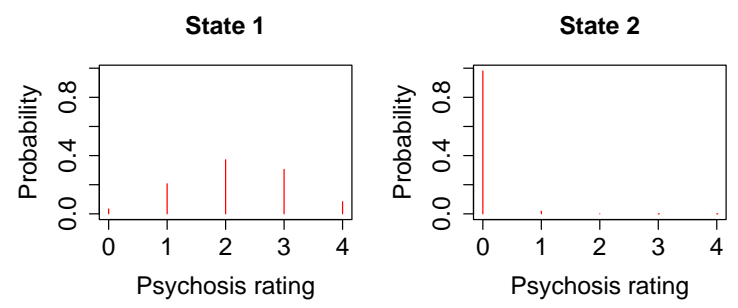

State 3

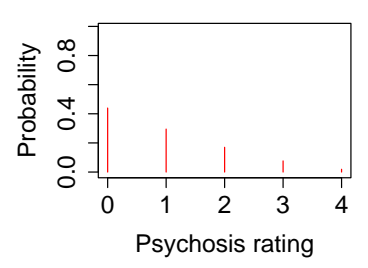

Figure 13: Marginal db distributions corresponding to each of the three states of a hidden Markov model fitted to the psychosis rating data.

\section{Appendix I}

Here I derive, from the conceptual definition (1) of the db distribution, an expression for the PMF of this distribution which, for fixed values of the support parameters $n_{\text {top }}$ and $\zeta$, is in exponential family form. A distribution is in the exponential family if its probability density or mass function has a particular structure. Different authors and books express this structure in a variety of equivalent ways. (See e.g., Cox and Hinkley 1974, p.94, Davidson 2003, p. 168, Hogg et al. 2005, p. 400. Liero and Zwanzig 2012, p. 15, Abramovich and Ritov 2013, p. 13. The reader may also find it useful to access https://en.wikipedia.org/wiki/Exponential_family.) Almost all of the commonly used distributions (with the notable exception of the uniform distribution) are in the exponential family.

A suitable expression for the exponential family form of a probability density or mass function, of a (scalar) distribution depending on a parameter vector $\boldsymbol{\theta}=\left(\theta_{1}, \ldots, \theta_{k}\right)^{\top}$, is

$$
f(x \mid \boldsymbol{\theta})=h(x) \exp \left(\sum_{i=1}^{k} \eta_{i}(\boldsymbol{\theta}) T_{i}(x)-A(\boldsymbol{\theta})\right) .
$$

The "natural parameters" of the distribution are the $\eta_{i}(\boldsymbol{\theta})$.

The pdf of the Beta distribution can be written in exponential family form (with natural parameters 
equal to $\alpha$ and $\beta$ ) as

$$
f(x \mid \alpha, \beta)=h_{\mathrm{B}}(x) \exp \{\alpha \log (x)+\beta \log (1-x)-\log B(\alpha, \beta)\},
$$

where $h_{\mathrm{B}}(x)=(x(1-x))^{-1}$, and $B(\alpha, \beta)$ is the beta function which is equal to $\Gamma(\alpha) \Gamma(\beta) / \Gamma(\alpha+\beta)$ (where in turn $\Gamma(\cdot)$ is the gamma function).

The PMF of the db distribution, expressed in terms of the Beta distribution (1), is

$$
\operatorname{Pr}(X=x \mid \alpha, \beta)=\frac{1}{\kappa} f\left(\frac{x-n_{\text {bot }}+1}{n_{\text {top }}-n_{\text {bot }}+2}\right),
$$

where $f(\cdot)$ is the pdf of the Beta distribution and

$$
\kappa=\sum_{i=n_{\mathrm{bot}}}^{n_{\mathrm{top}}} f\left(\frac{i-n_{\mathrm{bot}}+1}{n_{\mathrm{top}}-n_{\mathrm{bot}}+2}\right) .
$$

Hence, if one sets $h(x)=h_{\mathrm{B}}\left(\left(x-n_{\mathrm{bot}}+1\right) /\left(n_{\mathrm{top}}-n_{\mathrm{bot}}+2\right)\right), T_{1}(x)=\log \left(\left(x-n_{\mathrm{bot}}+1\right) /\left(n_{\mathrm{top}}-\right.\right.$ $\left.\left.n_{\text {bot }}+2\right)\right)$, and $T_{2}(x)=\log \left(\left(n_{\text {top }}-x+1\right) /\left(n_{\text {top }}-n_{\text {bot }}+2\right)\right.$, then the PMF of the db distribution can be written as

$$
\operatorname{Pr}(X=x \mid \alpha, \beta)=h(x) \exp \left\{\alpha T_{1}(x)+\beta T_{2}(x)-\log \left[\sum_{i=n_{\text {bot }}}^{n_{\text {top }}} h(i) \exp \left\{\alpha T_{1}(i)+\beta T_{2}(i)\right\}\right]\right\}
$$

Note that the $\log B(\alpha, \beta)$ terms, present in $f(\cdot)$, cancel when

$$
f\left(\frac{x-n_{\mathrm{bot}}+1}{n_{\mathrm{top}}-n_{\mathrm{bot}}+2}\right)
$$

is divided by $\kappa$.

The foregoing expression for the PMF is equal to

$$
\operatorname{Pr}(X=x \mid \alpha, \beta)=h(x) \exp \left\{\alpha T_{1}(x)+\beta T_{2}(x)-A(\alpha, \beta)\right\},
$$

where

$$
A(\alpha, \beta)=\log \left(\sum_{i=n_{\text {bot }}}^{n_{\text {top }}} h(i) \exp \left\{\alpha T_{1}(i)+\beta T_{2}(i)\right\}\right) .
$$

This is the expression given in (2) and is of exponential family form, although the constant $A(\alpha, \beta)$ might appear to be somewhat unorthodox. to 1 .

Obviously, the value of $A(\alpha, \beta)$ is such that the values of $\operatorname{Pr}(X=i \mid \alpha, \beta), i=n_{\mathrm{bot}}, \ldots, n_{\mathrm{top}}$, sum

\section{Appendix II}

When the PMF of a db distribution is expressed in the form (2), it is a relatively simple matter to derive an analytic expression for the gradient of the log-likelihood. Such an expression can be passed to optim() obviating the need for approximating the gradient numerically via finite differencing. The derivation of an analytic expression for the Hessian is equally easy. The optim() function makes no provision for using an analytically calculated Hessian. However, the availability of such an expression permits the calculation or estimation of the covariance matrix of the parameter estimates in an analytic manner. The derivations of the expressions for the gradient and Hessian are as follows.

The log-likelihood is

$$
\begin{aligned}
\ell & =\log \operatorname{Pr}\left(X=x \mid \alpha, \beta, n_{\mathrm{top}}, \zeta\right) \\
& =\log h(x)+\alpha T_{1}(x)+\beta T_{2}(x)-A(\alpha, \beta) .
\end{aligned}
$$

Consequently, the gradient is given by

$$
\frac{\partial \ell}{\partial \alpha}=T_{1}(x)-\frac{\partial A}{\partial \alpha} \quad \frac{\partial \ell}{\partial \beta}=T_{2}(x)-\frac{\partial A}{\partial \beta} .
$$


The Hessian is given by

$$
\begin{aligned}
\frac{\partial^{2} \ell}{\partial \alpha^{2}}=-\frac{\partial^{2} A}{\partial \alpha^{2}} & \frac{\partial^{2} \ell}{\partial \alpha \partial \beta}=-\frac{\partial^{2} A}{\partial \alpha \partial \beta} \\
\frac{\partial^{2} \ell}{\partial \beta^{2}} & =-\frac{\partial^{2} A}{\partial \beta^{2}}
\end{aligned}
$$

Now let

$$
E=\exp (A)=\sum_{i=n_{\text {bot }}}^{n_{\text {top }}} h(i) \exp \left\{\alpha T_{1}(i)+\beta T_{2}(i)\right\}
$$

Clearly

$$
\begin{aligned}
\frac{\partial A}{\partial \alpha}=\frac{1}{E} \frac{\partial E}{\partial \alpha} \frac{\partial A}{\partial \beta} & =\frac{1}{E} \frac{\partial E}{\partial \beta} \\
\frac{\partial^{2} A}{\partial \alpha^{2}}=\frac{1}{E} \frac{\partial^{2} E}{\partial \alpha^{2}}-\frac{1}{E^{2}}\left(\frac{\partial E}{\partial \alpha}\right)^{2} & \frac{\partial^{2} A}{\partial \alpha \partial \beta}=\frac{1}{E} \frac{\partial^{2} E}{\partial \alpha \partial \beta}-\frac{1}{E^{2}}\left(\frac{\partial E}{\partial \alpha} \frac{\partial E}{\partial \beta}\right) \\
\frac{\partial^{2} A}{\partial \beta^{2}} & =\frac{1}{E} \frac{\partial^{2} E}{\partial \beta^{2}}-\frac{1}{E^{2}}\left(\frac{\partial E}{\partial \beta}\right)^{2} .
\end{aligned}
$$

It remains to calculate the relevant partial derivatives of $E$. These are given by:

$$
\begin{aligned}
\frac{\partial E}{\partial \alpha} & =\sum_{i=n_{\text {bot }}}^{n_{\text {top }}} h(i) T_{1}(i) \exp \left(\alpha T_{1}(i)+\beta T_{2}(i)\right) \\
\frac{\partial E}{\partial \beta} & =\sum_{i=n_{\text {bot }}}^{n_{\text {top }}} h(i) T_{2}(i) \exp \left(\alpha T_{1}(i)+\beta T_{2}(i)\right) \\
\frac{\partial^{2} E}{\partial \alpha^{2}} & =\sum_{i=n_{\text {bot }}}^{n_{\text {top }}} h(i) T_{1}(i)^{2} \exp \left(\alpha T_{1}(i)+\beta T_{2}(i)\right) \\
\frac{\partial^{2} E}{\partial \alpha \partial \beta} & =\sum_{i=n_{\text {bot }}}^{n_{\text {top }}} h(i) T_{1}(i) T_{2}(i) \exp \left(\alpha T_{1}(i)+\beta T_{2}(i)\right) \\
\frac{\partial^{2} E}{\partial \beta^{2}} & =\sum_{i=n_{\text {bot }}}^{n_{\text {top }}} h(i) T_{2}(i)^{2} \exp \left(\alpha T_{1}(i)+\beta T_{2}(i)\right) .
\end{aligned}
$$

The foregoing calculations have been translated into $\mathrm{R}$ code in the (undocumented) functions $\operatorname{gradDb}()$ and hessDb() in the dbd package.

\section{Appendix III}

Covariance matrices of the maximum likelihood estimates of the parameters of a db distribution may be calculated both by theoretical means (using, e.g., aHess() from the dbd package) or by a Monte Carlo procedure using the mcCovMat () function from the same package. Doing such calculations in a number of examples has indicated that there can be noticeable discrepancies between the theoretical and Monte Carlo results. To investigate this issue further, I calculated the variance of $\hat{\beta}$ from known (rather than estimated) parameters using both the theoretical (inversion of the Fisher information matrix) and Monte Carlo procedures. The essential part of the code used to do this is as follows.

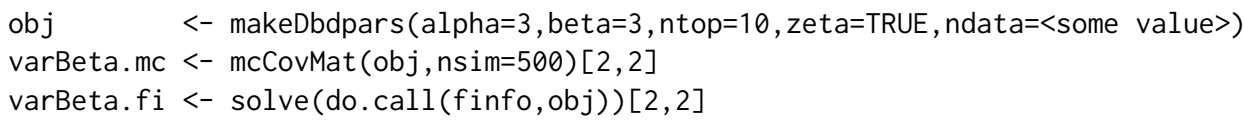

I effected the calculations for a range of sample sizes ("ndata"). The results are plotted in Figure 14.

The behavior depicted in Figure 14 is typical. The theoretical covariance matrices for the parameter estimates generally include variance entries which (for relatively small sample sizes) are appreciably smaller than the corresponding entries of the covariance matrices produced by Monte Carlo methods. Since the Monte Carlo covariance matrices are unbiased estimates of the true covariances, it would appear that the theoretical variances tend to underestimate the truth. This phenomenon is not peculiar to the $\mathrm{db}$ distribution. Such underestimation occurs in the context of the Beta distribution and very likely in other contexts as well. As illustrated by Figure 14, the level of underestimation (as would be expected) diminishes as the sample size increases. In the illustrated instance, the underestimation effectively disappeared when the sample size reached 200.

The fact that variances are underestimated by the theoretical covariance estimates implies that inference about the shape parameters based on the theoretical values should be treated with a certain amount of circumspection. Unless the sample size is large, confidence intervals may be somewhat too 


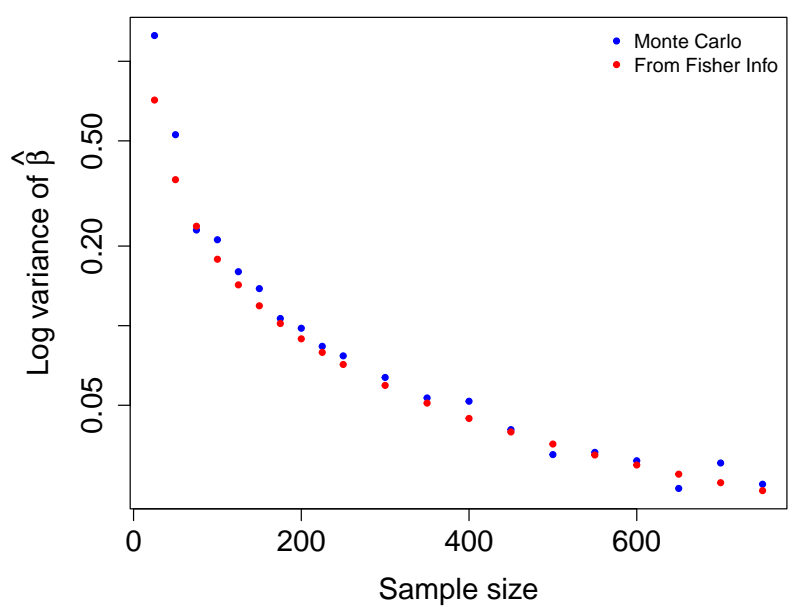

Figure 14: Monte Carlo and analytic estimates of the log variance of $\hat{\beta}$ for various sample sizes.

narrow, and hypothesis tests too liberal. When conducting inference about the shape parameters, it is advisable to estimate the covariance matrix using mcCovMat(). The procedure is not too computationally demanding and is thus reasonably quick in normal circumstances. It is probably a good idea, in circumstances in which inferential conclusions are critical, to calculate a number of Monte Carlo covariance matrix estimates (using different seeds) and to compare these with each other and with the "analytic" value of the covariance matrix.

The difference between results from using an "analytic" covariance matrix and those from using a Monte Carlo covariance matrix is also illustrated in Figure 15. The examples used are from the Bondi East Sydney Coliform Count data. (See Figures 10 and 11.) The chosen examples evince the most striking difference between the confidence ellipses based on the two different calculation methods.
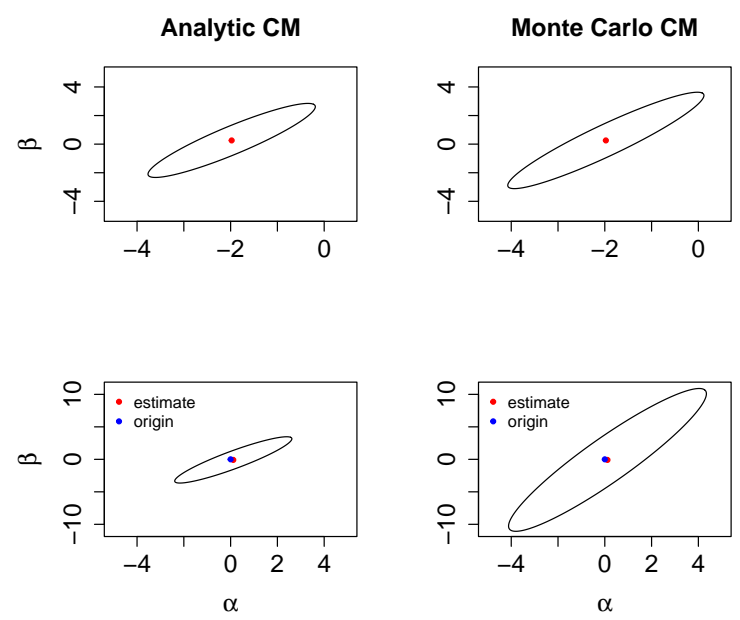

Figure 15: The top two panels show 95\% confidence ellipses for the parameters of the db distribution for the Bondi East data at depth equal to 40 meters. The left-hand panel is based on the "analytic" covariance matrix, the right-hand panel on a Monte Carlo covariance matrix. The bottom two panels show 95\% confidence ellipses for the difference in parameters between depth 60 meters and depth 40 meters. Again, the left-hand panel is based on the "analytic" covariance matrix and the right-hand panel on a Monte Carlo covariance matrix. The inferential conclusions with respect to the differences are unchanged. 


\section{Acknowledgements}

The author would like to thank an anonymous referee for useful comments which led to substantial improvements in the paper.

The author also wishes to acknowledge the use of the New Zealand eScience Infrastructure (NeSI) high-performance computing facilities, which made possible the analysis of the monocyte counts and psychosis ratings discussed in Example 5. New Zealand's national facilities are provided by NeSI and funded jointly by NeSI's collaborator institutions and through the Ministry of Business, Innovation \& Employment's Research Infrastructure programme. URL https://www. nesi .org.nz.

\section{Bibliography}

F. Abramovich and Y. Ritov. Statistical Theory: A Concise Introduction. CRC Press, Boca Raton, 2013. [p501]

M. Abramowitz and I. A. Stegun. Handbook of Mathematical Functions. Dover, New York, 1972. [p485]

A. Baddeley, E. Rubak, and R. Turner. Spatial Point Patterns: Methodology and Applications with R. Chapman and Hall/CRC Press, London, 2015. [p490]

D. R. Cox and D. V. Hinkley. Theoretical Statistics. Chapman and Hall, London, 1974. [p501]

A. C. Davidson. Statistical Models. Cambridge University Press, Cambridge, 2003. [p501]

A. Gandy and J. T. Kvaloy. Guaranteed conditional performance of control charts via bootstrap methods. Scandinavian Journal of Statistics, 40:647-668, 2013. doi: 10.1002/sjos.12006. [p486]

R. V. Hogg, J. W. McKean, and A. T. Craig. Introduction to Mathematical Statistics. Pearson/Prentice Hall, Upper Saddle River, New Jersey, 2005. [p501]

N. L. Johnson, S. Kotz, and N. Balakrishnan. Continuous Univariate Distributions, volume 2. Wiley, New York, 1995. [p485, 491]

H. Liero and S. Zwanzig. Introduction to the Theory of Statistical Inference. CRC Press, Boca Raton, 2012. [p501]

D. Murdoch and E. D. Chow. ellipse: Functions for Drawing Ellipses and Ellipse-Like Confidence Regions, 2018. URL https://CRAN. R-project.org/package=ellipse. R package version 0.4.1. [p494]

V. Parsonnet, D. Dean, and A. D. Bernstein. A method of uniform stratification of risk for evaluating the results of surgery in acquired adult heart disease. Circulation, 79:I3 - I12, 1989. [p486]

R Core Team. R: A Language and Environment for Statistical Computing. R Foundation for Statistical Computing, Vienna, Austria, 2020. URL https://www.R-project.org/. [p485]

J. G. Skellam. A probability distribution derived from the binomial distribution by regarding the probability of success as variable between the sets of trials. Journal of the Royal Statistical Society, Series B, 10:257-261, 1948. [p486]

S. H. Steiner, R. J. Cook, V. T. Farewell, and T. Treasure. Monitoring surgical performance using risk-adjusted cumulative sum charts. Biostatistics, 1:441 - 452, 2000. [p486]

B. Swihart and J. Lindsey. rmutil: Utilities for Nonlinear Regression and Repeated Measurements Models, 2020. URL https://CRAN. R-project.org/package=rmutil. R package version 1.1.4. [p486]

R. Turner. hmm.discnp: Hidden Markov models with discrete non-parametric observation distributions, 2020. R package version 3.0-6. [p485]

R. Turner. The db Distribution, 2021. R package version 0.0-22. [p489]

T. R. Turner, M. A. Cameron, and P. J. Thomson. Hidden Markov chains in generalized linear models. Canadian Journal of Statistics, 26:107 - 125, 1998. [p494]

C. H. Weiß. An Introduction to Discrete-Valued Time Series. John Wiley \& Sons, Chichester, 2018. [p493, 498] 
Rolf Turner

Honorary Research Fellow

Department of Statistics

The University of Auckland

New Zealand

ORCID: 0000-0001-5521-5218

r. turner@auckland. ac.nz 